\title{
Freezers, Dry Ice and Phase-Change Materials for Cold Chain Equipment in Ultra Low Temperature Logistics
}

\author{
Amelia Carolina Sparavigna \\ Department of Applied Science and Technology, Politecnico di Torino, Torino, Italy \\ Email: amelia.sparavigna@polito.it
}

Torino $22 / 05 / 2021$

\begin{abstract}
Freezers and dry ice are used for cold chains. Besides them, phase change materials (PCMs) are also involved. A PCM is a substance absorbing and releasing energy at phase transition. The substance is therefore useful for heating and cooling applications. Here we review some facts about the use of freezer, dry-ice and PCMs for equipment in Ultra Low Temperature Logistics.
\end{abstract}

Keywords: Freezers, Dry Ice, PCM, PCC, Composite Materials, Phase Change Composites, Thermal Management, Latent Heat Storage, Ultra Low Temperature Logistics, Cold Chain.

Subject Areas: Materials Science, Logistics.

\section{Introduction}

Freezers are fundamental equipment for cold chains, such as dry ice is largely used for the Ultra Low Temperature (ULT) shipping. Besides them, phase change materials (PCMs) are also involved. A PCM is a substance absorbing and releasing energy at phase transition. The substance is therefore useful for heating and cooling applications. The most used transition is that between solid and liquid phases. Then, we can immediately recognize that PCM are latent heat storage (LHS) materials and that the most common phase change material is the ice (heat of fusion, $333.55 \mathrm{~J} / \mathrm{g}$ ). Ice can be used to store winter cold to cool buildings in summer [1].

According to the modern classification, a phase change which is involving latent heat is a firstorder phase transition. During such a transition, the material either absorbs or releases some amount of energy per volume. The temperature of the thermodynamic system remains constant when heat is added or subtracted, and the system consists of a mixture of the two phases. Let us also remember that the phenomenon of supercooling exists. Supercooling is the process of lowering the temperature of a liquid, below its freezing point, without it becoming solid. It happens in the absence of nucleating agents, around which a crystal structure can form. 
In the last few decades, several substances have been developed and adapted to have further ap plications to a quite wide temperature range. Today, we can find several PCMs used in passive freezers and mentioned by the World Health Organization, WHO, in its logistic guidance for COVID-19 vaccination [2]. These PCMs are used in the vaccination ULT logistics, where thermal energy storage is fundamental. In general, the ULT logistics takes advantage of the fast progress in materials science, which is providing new substances for PCMs, and also from new technological equipment.

Among the materials used for cooling, we can find dry ice largely used. It is generally involved for packaging and transporting food and pharmaceutical products (such as vaccines) and biological samples, needing to be kept frozen. Actually, dry ice is a very effective coolant. How ever, this material is dangerous, if not handled with care. The sublimation produces carbon dioxide vapors, which is harmful in the case of high concentrations and besides the fact of enhancing carbon emissions in atmosphere. Phase Change Materials can substitute dry ice.

\section{ULT equipment and Cold Chain Logistics}

A cold chain is a temperature-controlled supply chain. When unbroken, a cold chain consists in a series of refrigerated production, storage and distribution of goods, requiring specific equipment and logistics. The cold chain maintains the quality of the products via a specifically controlled low-temperature range. Such a chain is required, among others, by many pharmaceutical products, such as vaccines. Among them, m-RNA vaccines are requiring the Ultra Low Temperature (ULT) chain [2].

Cold chains need, besides specific equipment in production and primary storage sites, low temperature devices and related packaging methods for transportation and transient storage. Adequate packaging and cold storage can be crucial to prevent quantitative and qualitative losses of products. Therefore sensors, data-loggers and related technologies are required for monitoring the temperature of products.

ULT cold storage is usually referring to a thermally environment kept at or below $-20^{\circ} \mathrm{C}$. In many instances, the devices used to achieve the required temperatures are freezers. ULT freezer temperatures typically range between $-40^{\circ} \mathrm{C}$ to $-86^{\circ} \mathrm{C}\left(-40^{\circ} \mathrm{F}\right.$ to $\left.-122.8^{\circ} \mathrm{F}\right)$. Like the household refrigeration units, ULT freezers can come in devices having several forms: upright, chest, undercounter/benchtop and also portable models. Compressor-based ULT freezers achieve ultracold temperatures with two compressors and cooling loops in a cascading arrangement.

Ultra-low temperature (ULT) freezers are therefore required by any organization which handles temperature-sensitive specimens and therapies, besides mRNA vaccines. In fact, an ULT freezer is a cold storage equipment fundamental for the cold chain of pharmaceutical, healthcare and biotechnological industries [3].

\section{ULT portable equipment and last-mile}

Ref.[3] gives some information to understand and evaluate freezer technology for the ULT transportation. What are the advantages of ULT freezers, compared to alternatives, such as dry ice? This is a question that we can find in [3]. Advantages are a precise and reliable temperature control in a wider temperature range, with integration of a secondary remote temperature 
monitoring. Only personal protective equipment needed are commonly available gloves, and no significant personnel risks exist or ventilation requirements. From a logistics standpoint - it is noted in [3] - ULT freezers are the best way to preserve the cold chain long-term. In fact, the use of dry ice is not a permanent solution, and it is requiring a regular refill and monitoring to maintain the temperature set-point.

Another question posed in [3] is the following: how is it possible to maintain vaccine efficacy and the cold chain during last-mile delivery and patient point of use? "One of the most significant challenges of the Coronavirus disease (COVID-19) vaccine administration is maintaining safe and effective doses, on-site, over extended periods of time in local health agencies, clinics and pharmacies where vaccines are typically administered". However, as noted in [3], ULT freezers are not the usual equipment of these facilities. Large ULT freezers are too expense, besides being bulky and noisy. And dry ice is not providing a long-term temperature stability. However, small and portable ULT freezers, that were designed for use in small, rural or remote locations, exist and can be easily moved throughout clinical sites. Moreover, the use of small ULT freezers can also help "to avoid last mile cold storage bottlenecks and get patients vaccinated faster".

Ref. [3] concludes stressing that ULT freezers are ultracold equipment that will become integral in all the phases "of the coming mRNA technology revolution. Advancements in cell and gene therapy, regenerative medicine, vaccines and immunotherapies will most likely require ULT freezers to safely preserve priceless biological materials". This revolution is based on materials having a limited life span and requires low temperature control to avoid degradation. It is probably one of the main aspects of the future of medicine, involving pharmaceutical and vaccine manufacturers, third-party logistics providers and clinical point-of-care facilities, all requiring the cold storage capacity.

\section{ULT passive equipment for shipping vaccines}

In [2], passive equipment (ULT insulated containers) for shipping vaccines are discussed. "When selecting which passive container to use, consider the storage temperature and duration of storage". There are two types of passive equipment: Arktek and Thermal Shippers.

Arktek. It is a super-insulated, double-walled large bottle-like container that uses multilayer insulation technology and eight PCM packs to keep vaccines at ULTs $\left(-80^{\circ} \mathrm{C}\right.$ to $\left.-60{ }^{\circ} \mathrm{C}\right)$ in remote storage and vaccination sites for up to 5 days without any powered refrigeration or extra coolant. - Thermal shippers. They are shippers used by manufacturers to ship vaccines. Like the Arktek, they do not need an external power supply. They use a combination of insulation technology and coolants (dry ice for instance) to keep vaccines at ULTs $\left(-80{ }^{\circ} \mathrm{C}\right.$ to $\left.-60{ }^{\circ} \mathrm{C}\right)$ over the short term. Typically, they possess an impact-resistant outer layer. Thermal shippers come in a range of sizes. These shippers can also be reused, with proper cautions, for longer than 5 days, in the case that dry ice is regularly replenished.

The passive equipment need coolant/freezing materials. There are two primary coolant materials that can be used: Special ULT PCM and Dry Ice.

PCMs are used for passive freezing when transporting and temporarily storing vaccines in ULT insulated containers. For Pfizer vaccine, PCMs have melting point of $-78^{\circ} \mathrm{C}$ to $-65^{\circ} \mathrm{C}$, which is within the required vaccine storage temperatures range of $-80{ }^{\circ} \mathrm{C}$ to $-60{ }^{\circ} \mathrm{C}$. In [2], the $\mathrm{PCm}$ 
which is mentioned is PlusICE E-78. It contains a solid nucleating agent. Without this agent, the liquid will not freeze even when cooled below $-90{ }^{\circ} \mathrm{C}$.

Dry ice or frozen carbon dioxide $\left(\mathrm{CO}_{2}\right)$ has a phase change temperature of $-78{ }^{\circ} \mathrm{C}$ and can also maintain vaccine storage temperature less than $-60{ }^{\circ} \mathrm{C}$. "Dry ice can either be produced with a dry ice machine or procured from local suppliers. Dry ice is recommended for use with thermal shippers since its phase change takes longer than PCM. Dry ice and special ULT PCMs are used for UCC (Ultra Cold Chain) equipment as coolant/freezing packs" [2].

\section{Softbox supporting Pfizer}

In [4-5], Softbox, which is a global provider of solutions for a passive temperature control packaging, illustrates how it is supporting Pfizer in the distribution of COVID-19 vaccines. It is providing a high-performance, temperature-controlled, parcel shipper for ULT applications. "Ultra-Low Temperature vaccines, such as BioNTech, ... use mRNA (messenger RNA) technology and must be stored at temperatures between $-90^{\circ} \mathrm{C}$ to $-60^{\circ} \mathrm{C}$ to ensure that the vaccine's quality and efficacy is maintained. The ULT shipper is reportedly capable of maintaining the required temperature during the shipping of COVID- 19 vaccines between $-90^{\circ} \mathrm{C}$ to $-60^{\circ} \mathrm{C}$ for at least 10 days unopened". For this shipping solution, there is required the use high-performance insulation materials in conjunction with dry ice. "Softbox ULT Shipper can be opened twice a day, for up to three minutes at a time. This allows clinicians at Point of Use (POU) sites to access the vaccine vials required for each day's immunisation clinics without exposing the remaining vaccine stored within the shipper to ambient temperatures, thus ensuring the integrity of the vaccine is maintained ... When correctly managed, the ULT Shipper can be used to store vaccines for in excess of 30 days". By means of "Re-Icing", the dry ice in Softbox ULT Shippers "can be topped up, ensuring maximum thermal protection".

\begin{tabular}{|l|l|l|l|l|l|}
\hline Material & Supplier & Type & \multicolumn{1}{|l|}{ Form } & $\begin{array}{l}\text { Melting } \\
\text { point, } \\
\mathrm{T}_{\mathrm{m}}\end{array}$ & $\begin{array}{c}\text { Heat } \\
\text { of } \\
\text { fusion, } \\
\mathbf{k J} / \mathbf{k g}\end{array}$ \\
\hline E-114 & PlusICE & Eutectic & Bulk & $\begin{array}{l}-114^{\circ} \mathrm{C} \\
\left(-173^{\circ} \mathrm{F}\right)\end{array}$ & 107 \\
\hline E-90 & PlusICE & Eutectic & Bulk & $\begin{array}{l}-90^{\circ} \mathrm{C} \\
\left(-130^{\circ} \mathrm{F}\right)\end{array}$ & 90 \\
\hline E-78 & PlusICE & Eutectic & Bulk & $\begin{array}{l}-78^{\circ} \mathrm{C} \\
\left(-108^{\circ} \mathrm{F}\right)\end{array}$ & 115 \\
\hline E-75 & PlusICE & Eutectic & Bulk & $\begin{array}{l}-75^{\circ} \mathrm{C} \\
\left(-103^{\circ} \mathrm{F}\right)\end{array}$ & 102 \\
\hline
\end{tabular}

\section{PCM instead of dry ice}

As we have seen before, for ULT shipping we need the proper packaging, made of thermal insulating materials and dry-ice inside. Dry ice sublimates at $194.7 \mathrm{~K}\left(-78.5^{\circ} \mathrm{C} ;-109.2{ }^{\circ} \mathrm{F}\right)$ at at mospheric pressure. Commercially PCMs for this temperature are available (listed by 
Wikipedia), the data of which are given in the previous table. E-78 PlusICE is mentioned in [2].

According to their chemical composition, PCMs are usually divided into three types: Organic PCMs (fatty acids and paraffin wax), inorganic PCMs (salts, hydrated salts and metallics), and eutectic PCMs. As we can see from the Table in Wikipedia, PCMs for ULTs, such as the E-78 PlusICE, are eutectic mixtures.

One type of organic PCM is the paraffin. The melting-point temperature of paraffin can be changed by tailoring the length of its hydrocarbon chain. Increasing the length of the chain means an increase of melting-point temperature. The non-paraffin PCM category includes fatty acids, glycols, alcohols, and esters [6]. The inorganic PCMs are materials which are not carbon-based. They comprise a wide range of materials (water, hydrated salts, low-melting-point metals) [6]. Of these, water and the hydrated salts are the most useful for Cold Chain Equipment (CCE). "Water is a great inorganic PCM from many perspectives. It has a very high latent heat; has higher density than many of the organic PCMs; is stable, nontoxic, readily available, and cheap; and expands only moderately during freezing. However, water's phase change occurs at $0^{\circ} \mathrm{C}$, the very low end of the desirable range for CCE, and water is prone to supercooling, making it less than ideal for vaccine freeze-prevention applications" [6]. The eutectic PCMs consist of combinations of compounds, which may be organic, inorganic, or organic and inorganic compounds. "Melting/freezing of the two components of the eutectic occurs simultaneously (congruent melting/freezing)" [6].

\section{Eutectic systems}

Eutectic systems (their name from Greek "عv́", eu = well, and " $\tau \dot{\eta} \xi ı "$ ", tēxis = melting) is a mixture of substances that melts or solidifies at a temperature which is lower than the melting point of any of the constituents [7]. This temperature is the eutectic temperature, and is the lowest possible melting temperature over all of the mixing ratios for the involved component species.

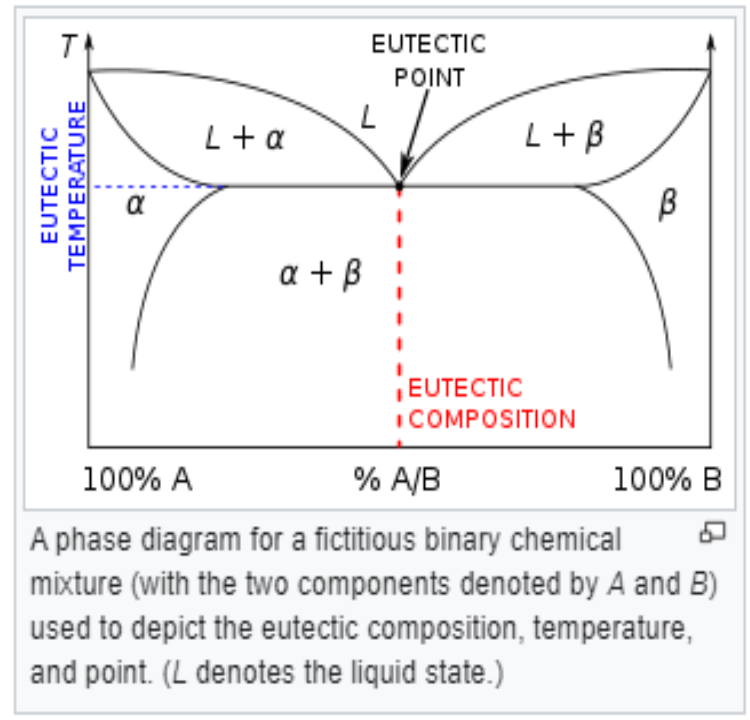

The diagram is from Wikipedia, en.wikipedia.org/wiki/Eutectic_system

A non-eutectic mixture, that is a mixture having a ratio of components different from that of the eutectic one, has a different melting temperature for its different constituents, as shown by the diagram. And also, as a non-eutectic mixture cools down, each of its components would solidify at a different temperature, until the entire mass is solid. 
Sodium chloride and water possesses the eutectic mixture having eutectic point at $-21.2{ }^{\circ} \mathrm{C}$ [8] and $23.3 \%$ salt by mass [9]. "Solar salt", $60 \% \mathrm{NaNO}_{3}$ and $40 \% \mathrm{KNO}_{3}$, forms eutectic molten salt mixture which is used for thermal energy storage in concentrated solar power plants [10].

\section{Avoiding supercooling}

In [2], it is told that PCM PlusICE E-78 contains "solid nucleating agent, without which the liquid will supercool substantially and will not freeze even when cooled below $-90^{\circ} \mathrm{C}$ ". Let us discuss the supercooling phenomenon as in [6]. "Kinetic characteristics include supercooling and the rate of phase change. When a PCM undergoes supercooling, it cools below the phasechange temperature and then rapidly undergoes the phase change. "For example, under some conditions, if water is cooled slowly and left undisturbed, it may cool several degrees below $0^{\circ} \mathrm{C}$ before beginning to freeze". "The tendency for supercooling can be reduced by the addition of nucleation particles to a PCM or by careful PCM container design".

"For a PCM to work efficiently, the difference between the melting temperature and solidification temperature should be as low as possible. However, in most of the PCMs, it is observed that solidification starts at a temperature much below the melting point. This phenomenon, known as sub-cooling, leads to significant loss in the storage/retrieval of latent energy which is undesirable" [11]. In [11], we can find a review of the various methods, investigated "to overcome the problems associated with sub-cooling. The various methods discussed include - addition of appropriate nucleating agents of similar crystal structure as that of the PCM, cold finger technique and application of surface roughness on the walls containing the PCM, ultrasonic irradiation, application of dynamic pressure, cavitation and electrical means".

\section{Encapsulation}

As observed in [6], besides the PCM itself, it is necessary to consider how it is contained. A PCM can corrode, penetrate or dissolve the material of its container. Moreover, large-volume containers can be thermally inefficient, because the heat transfer through the PCM is resulting too slow [6]. Therefore, an efficient package needs a high surface area-to-volume ratio. An alternative is that of incorporating fins to transfer heat quickly into the bulk [6].

"A particular type of PCM packaging allowing novel applications is encapsulation. PCMs may come encapsulated in polymer spheres ranging from macroscopic to microscopic. Encapsulated PCMs may then be incorporated into fibers or compressed together into blocks. PCM encapsulation can improve PCM performance and stability by reducing phase separation during freezing and melting and protecting the PCM from chemical or physical degradation. ... However, use of encapsulation can decrease the effective density of a PCM and thus require larger volumes of encapsulated PCMs than the non-encapsulated PCM. The addition of encapsulation material also affects disposal considerations. For example, a biodegradable PCM that is encapsulated in a non-biodegradable plastic becomes a non-biodegradable waste" [6]. We have observed that, recently, researches for encapsulation of PCM by biochar, an ecologic material, have been proposed [12]. 


\section{References}

[1] Sparavigna, A. C., Giurdanella, S., \& Patrucco, M. (2011). Behaviour of Thermodynamic Models with Phase Change Materials under Periodic Conditions. Energy and Power Engineering, 3(02), 150. DOI: 10.4236/epe.2011.32019

[2] COVID-19 vaccination: supply and logistics guidance. WHO. Feb 12, 2021.

[3] Scott Masiella (March 14, 2021). The Opportunities and Obstacles of Ultra-Low Temperature Storage in Pharmaceutical Cold Chain Logistics Stirling Ultracold - https://www.sdcexec.com/transportation/cold-chain/article/21295337/stirling-ultracold-the-opportunitiesand-obstacles-of-ultralow-temperature-storage-in-pharmaceutical-cold-chain-logistics

[4] packagingeurope.com/softbox-supports-pfizer-in-global-cold-chain-distribution-ofCOVID-vaccine/

[5] www.healthcarepackaging.com/logistics-distribution/cold-chain-temperaturecontrol/news/21354870/softbox-supports-pfizer-in-the-global-cold-chain-distribution-ofcovid19-vaccines

[6] P. Lennon. 2016. Investigation of PhaseChange Materials for Vaccine Cold Chain Equipment, https://path.azureedge.net/media/documents/DT pcm rpt1.pdf

[7] Guthrie, F. (1884). "On Eutexia". Physical Society: 462-482.

[8] Muldrew, Ken; Locksley E. McGann (1997). Phase Diagrams. Cryobiology-A Short Course. University of Calgary.

[9] Senese, Fred (1999). Does salt water expand as much as fresh water does when it freezes?. Solutions: Frequently asked questions. Department of Chemistry, Frostburg State University.

[10] Molten salts properties. Archimede Solar Plant Specs.

[11] Kashyap G. Rao, Pavankumar Rasoor, G. Anjaneya, J. R. Nataraj, and M. R. Srinivas. A review on methods of preventing super cooling in phase change materials (PCMs). AIP Conference Proceedings 2317, 020003 (2021). DOI: 10.1063/5.0036177

[12] Sparavigna, A. C., Phase-Change Materials and Biochar: Some New Composite Materials in Recent Literature (May 18, 2021). SSRN. DOI: 10.2139/ssrn.3848357 